\title{
Editorial
}

\section{Vancomycin-Intermediate Staphylococcus epidermidis: Curio or Omen?}

\author{
Larry J. Strausbaugh, MD
}

In this issue, Garrett and colleagues report a case of bloodstream infection caused by a strain of Staphylococcus epidermidis that exhibited reduced susceptibility to vancomycin. ${ }^{1}$ Two isolates, collected on different days, were tested against vancomycin in three different laboratories. In disk-diffusion assays, both isolates appeared to be susceptible to vancomycin. However, tests using the Microscan Walk-Away System (MicroScan, West Sacramento, CA) yielded minimum inhibitory concentrations (MICs) of 16 $\mu \mathrm{g} / \mathrm{mL}$. E-tests and broth microdilution assays yielded MICs of $8 \mu \mathrm{g} / \mathrm{mL}$. Both values fall within the intermediate range for vancomycin. Strains of $S$ epidermidis with MICs $\leqslant 4 \mu \mathrm{g} / \mathrm{mL}$ are considered susceptible, and strains with MICs $\geqslant 32 \mu \mathrm{g} / \mathrm{mL}$ are considered resistant. ${ }^{2}$ Strains with values in the intermediate range are variably said to have decreased susceptibility, intermediate resistance, or increased resistance. The term vancomycin-intermediate $S$ epidermidis, or VISE, is appealing because of its consistency with the terminology used for the original isolates of Staphylococcus aureus with similar MICs.,4 Regardless of the terminology, the basic phenomenon is new to the clinical microbiology laboratory.

Three features of the reported case suggest that these intermediate MIC values are more than laboratory anomalies. First, the patient had received almost 40 days of vancomycin therapy prior to developing bacteremia caused by the $S$ epidermidis isolate with reduced susceptibility. Prolonged exposure to vancomycin and the presence of an indwelling central venous catheter probably constitute an ideal setting for the development of resistance. Second, the patient was symptomatic at the time of the bacteremia, and the isolate was recovered from specimens obtained through both the Groshong central venous catheter and a peripheral vein. These details unequivocally establish the clinical diagnosis of infection. Lastly, the bacterium was reisolated from both sites 5 days later, despite ongoing therapy with vancomycin. Because the central venous catheter was sterile when it was removed 2 days later, persistence of bacteremia for 5 days seems unusual. This sluggish response suggests that the therapeutic power of vancomycin was impaired or reduced; however, this suggestion requires the caveat that serum concentrations of vancomycin were not reported.

What are hospital epidemiologists and others to make of this case report? Is the observation a curio or noveltyjust "one of those things" that soon will be forgotten? Or is it an omen, a harbinger of things to come? Although no one can be certain at this point, several lines of evidence suggest that this case is a warning of more to come. Of note, cases of infection caused by $S$ epidermidis with reduced susceptibility or frank resistance to vancomycin have been reported from Europe during the last several years. ${ }^{57}$ In one report from the United Kingdom, a patient developed peritonitis associated with chronic ambulatory peritoneal dialysis (CAPD) after receiving repeated courses of vancomycin. ${ }^{6}$ Two different strains of $S$ epidermidis isolated from this individual had vancomycin MICs of 8 and $32 \mu \mathrm{g} / \mathrm{mL}$, respectively. Another report from the Slovak Republic described four cancer patients with catheter-associated bloodstream infections caused by strains of $S$ epidermidis with vancomycin MICs of $16 \mu \mathrm{g} / \mathrm{mL}^{7}$ Central venous catheters, neutropenia, prophylaxis with ofloxacin, and prior vancomycin therapy were common antecedents in these four patients.

Multiple sightings of $S$ epidermidis isolates with decreased susceptibility suggest the arrival of an important trend. Such a trend is not unexpected in light of the increasing prevalence of nosocomial infections caused by coagulasenegative staphylococci and the corresponding increase in vancomycin use to treat them. Moreover, all of the reported cases have arisen in the logical setting, with prior vancomycin therapy and indwelling prosthetic devices. 
Resistance in $S$ epidermidis is emerging at a time when the relationship between vancomycin and other Staphylococcus species appears stressed. One thinks first of $S$ aureus strains with reduced susceptibility to vancomycin that have been recovered from patients during the last 2 years in Japan, Michigan, New Jersey, and New York. $3,4,8,9$ These strains have demonstrated vancomycin MICs of $8 \mu \mathrm{g} / \mathrm{mL}$.

Additionally, strains of Staphylococcus haemolyticus have demonstrated resistance to vancomycin. A study of coagulase-negative staphylococci undertaken more than 10 years ago at two hospitals in Virginia indicated that $13 \%$ of their 524 clinical isolates were $S$ haemolyticus and that $62 \%$ of $47 S$ haemolyticus strains tested against vancomycin had MICs $\geqslant 6.25 \mu \mathrm{g} / \mathrm{mL} .{ }^{10}$ One case report from North Carolina described repeated episodes of peritonitis in the setting of CAPD caused by strains of $S$ haemolyticus demonstrating a stepwise increase in vancomycin MICs. ${ }^{11}$ The strains isolated late in the course had MICs of $8 \mu \mathrm{g} / \mathrm{mL}$. Similarly, another case report described bacteremia caused by an isolate of this bacterium with MICs of 8 to $16 \mu \mathrm{g} / \mathrm{mL} .{ }^{12}$ It was obtained from a patient in Iowa who had acute leukemia and had received multiple antibiotics, including vancomycin, through a Hickman catheter during the preceding month. Closely related isolates obtained from the patient before the bacteremia occurred had MICs of 1.0 to $2.0 \mu \mathrm{g} / \mathrm{mL}$, indicating that resistance developed in association with prolonged empirical vancomycin therapy. All isolates from this latter case developed MICs of $32 \mu \mathrm{g} / \mathrm{mL}$ after serial passage through increasing concentrations of vancomycin in vitro. Other investigators have selected for vancomycin resistance in vitro in clinical isolates of $S$ haemolyticus. ${ }^{13}$ Taken as a whole, these observations suggest that staphylococcal resistance to vancomycin may continue to crop up and may intensify in different species and in diverse locales.

Observations about teicoplanin are also germane to this discussion. Teicoplanin, another glycopeptide antibiotic, is similar to vancomycin in structure and potency. It has been used in several European countries for almost a decade. In the laboratory, stepwise exposure of $S$ epider- . midis strains to increasing concentrations of teicoplanin generates resistance..$^{14}$ Not surprisingly, teicoplanin resistance has been found with increasing frequency in clinical isolates of staphylococci. Results from one French study indicated that $23.2 \%$ of 362 strains of coagulase-negative staphylococci collected in 1988 and 1989 were intermediate and $1.7 \%$ were resistant to teicoplanin. ${ }^{15}$ Resistant strains belonged to the species of $S$ epidermidis (74\%) and $S$ haemolyticus (19\%). In another French study from the same period, teicoplanin resistance was correlated with prior receipt of teicoplanin or vancomycin. ${ }^{16}$ Only $20 \%$ of isolates from patients who had not received prior glycopeptide therapy were intermediate or resistant to teicoplanin. In contrast, $49.2 \%$ of strains from patients who previously had received glycopeptides were intermediate or resistant.

Minimum inhibitory concentrations of teicoplanin for coagulase-negative staphylococci in the intermediate and resistant range also have been reported in the United States. ${ }^{10,17,18} S$ epidermidis and $S$ haemolyticus are the species accounting for most of the strains with decreased susceptibility to teicoplanin. In a recently published study from the United States, 28 of 41 strains of $S$ epidermidis and $S$ haemolyticus exhibited tube-dilution teicoplanin MICs of 4 to $8 \mu \mathrm{g} / \mathrm{mL}$, which increased to 16 to $32 \mu \mathrm{g} / \mathrm{mL}$ upon prolonged incubation. ${ }^{18}$ Cultures of the bacteria with reduced susceptibility were heterogeneous. They contained subpopulations with frequencies of $10^{-5}$ to $10^{-4}$ that could grow on up to $50 \mu \mathrm{g} / \mathrm{mL}$ of teicoplanin. Cultures of the same bacteria also were heterogeneous for vancomycin resistance. Subpopulations present at frequencies of $10^{-5}$ to $10^{-7}$ could grow in media with 6 to $12 \mu \mathrm{g} / \mathrm{mL}$ of vancomycin. Enrichment of cultures for the resistant subpopulations was said to have ". . . occurred with relative ease under laboratory conditions." 18

Treatment results with teicoplanin for staphylococcal infections have been generally favorable and comparable to those achieved with vancomycin in comparative trials. ${ }^{19}$ However, a case report from Kaatz and colleagues, which described a patient with endocarditis caused by $S$ aureus who received teicoplanin, documented the emergence of resistance during therapy ${ }^{20}$ Sequential isolates recovered from blood cultures during the first 10 days of therapy exhibited increasing MICs. The MIC of teicoplanin for the isolate obtained on admission was $1.0 \mu \mathrm{g} / \mathrm{mL}$, whereas the MIC for the isolate obtained on hospital day 10 was 8.0 $\mu \mathrm{g} / \mathrm{mL}$. Resistance was maintained in the laboratory, despite serial passage on drug-free agar. Exposure of the parent strain to teicoplanin in vitro yielded resistant subpopulations at frequencies of $10^{-7}$ to $10^{-9}$. Evaluation of 18 other strains of $S$ aureus from diverse locations identified the emergence of similar subpopulations after exposure to teicoplanin in the laboratory. Teicoplanin induced resistance in these strains more readily than vancomycin.

The basis of glycopeptide resistance in staphylococci is unknown, but it appears to differ substantially from that observed in enterococci. Specifically, assays for vanA, $\operatorname{van} \mathrm{B}$, and $\operatorname{van} \mathrm{C}$ genes have been negative. ${ }^{1,8,21}$ Evaluation of the $S$ aureus isolates recovered by Kaatz and coworkers have yielded insights concerning the possible mechanism(s) of resistance. Shlaes et al provided evidence suggesting that the resistant strain expresses a cell wall that differs in structure from that of its susceptible parent. ${ }^{22,23}$ The resistant strains demonstrate increased expression of the two polypeptides of penicillin-binding protein 2 (PBP2), expression of a new $35-\mathrm{kd}$ membrane protein, and increased susceptibility to lysis by lysostaphin, an endopeptidase that cleaves the cross-link of the staphylococcal cellwall peptidoglycan. These investigators generated additional data indicating that a minimum of two mutational steps could produce vancomycin resistance in these strains of $S$ aureus. ${ }^{23}$ Others have correlated glycopeptide resistance in $S$ aureus with increased expression of PBP2 and have suggested that increased concentrations of PBP2 might compete with glycopeptide binding to the D-AlanylD-Alanine terminus of the peptidoglycan precursors. ${ }^{8,24}$ Interestingly, studies with resistant strains of $S$ epidermidis have demonstrated abnormally thickened cell walls, a find- 
ing attributed to overproduction of glycopeptide binding sites within the cell-wall peptidoglycan. ${ }^{25}$ Clearly, more work is needed to clarify the mechanism (s) of glycopeptide resistance in staphylococci; in fact, it should be viewed as a high priority to advance new drug development.

Another priority suggested by the report from Garrett and colleagues is enhanced surveillance. Glycopeptide resistance in staphylococci may be more prevalent than is realized currently. Available data suggest where to look. Surveillance should extend to strains of coagulase-negative staphylococci, as well as to strains of $S$ aureus. Isolates from CAPD patients, neutropenic cancer patients, and others with invasive devices who have received prolonged or repeated courses of vancomycin therapy deserve the closest scrutiny. As indicated in the current report and elsewhere, disk-diffusion assays may not be sufficient to detect this form of resistance. ${ }^{21}$ Nonautomated quantitative tests using 24-hour incubation periods are the most accurate methods for detecting staphylococci with reduced susceptibility to glycopeptide antibiotics. ${ }^{21}$

A third priority is continuing study of the epidemiology as new cases appear. Although nasal cultures of contacts were negative for the $S$ epidermidis isolate with reduced susceptibility to vancomycin in their study, one wonders if one specimen from the nose and the bacteriologic methods used were sufficient to exclude nosocomial spread. Given the presence of innumerable S epidermidis on the skin of healthy caregivers, detecting a resistant bacterium may be likened to finding the proverbial needle in the haystack, especially if it was present only transiently. In the future, investigators may wish to obtain multiple specimens from different cutaneous sites, including the hands. They also may wish to use methods that maximize detection of resistant strains, eg, direct plating of specimens on agar plates containing vancomycin or plating large inocula derived from broth cultures of original specimens to vancomycin-containing agar. The utility of such methods in defining the source of methicillin-resistant coagulase-negative staphylococci at surgical sites illustrates their potential value in this area of investigation. ${ }^{26}$ Obviously, information about the epidemiology of resistant strains will be much desired to formulate guidelines for prevention of nosocomial transmission.

In summary, I think that this first report from the United States of an infection caused by $S$ epidermidis with decreased susceptibility to vancomycin is much more than happenstance. Given the rumblings among the staphylococci noted above, I view this report as a warning that more glycopeptide resistance in staphylococci is on the way. I think that we can bet on seeing more of it in the future. (Has anyone lost such a bet in recent years?) Accordingly, I would urge that attention be given to the priorities outlined above.

\section{REFERENCES}

1. Garrett DO, Jochimsen E, Murfitt K, Hill B, McAllister S, Nelson P, et al. The emergence of decreased susceptibility to vancomycin in Staphylococcus epidermidis. Infect Control Hosp Epidemiol 1999;20:167-170.

2. National Committee for Clinical Laboratory Standards. Performance Standards for Antimicrobial Susceptibility Testing; Eighth Informational Supplement. NCCIS document M100-S8. Wayne, PA: NCCLS; 1998.
3. Centers for Disease Control and Prevention. Staphylococcus aureus with reduced susceptibility to vancomycin-United States, 1997. MMWR 1997;46:765-766.

4. Centers for Disease Control and Prevention. Update: Staphylococcus aureus with reduced susceptibility to vancomycin-United States, 1997. MMWR 1997;46:813-815.

5. Sanyal D, Johnson AP, George RC, Cookson BD, Williams AJ. Peritonitis due to vancomycin-resistant Staphylococcus epidermidis. Lancet $1991 ; 337: 54$

6. Sanyal D, Johnson AP, George RC, Edwards R, Greenwood D. In-vitro characteristics of glycopeptide resistant strains of Staphylococcus epidermidis isolated from patients on CAPD. I Antimicrob Chemother 1993;32:267-278.

7. Kremery V Jr, Trupl J, Drgona L, Lacka J, Kukuckova E, Oravcova E. Nosocomial bacteremia due to vancomycin-resistant Staphylococcus epidermidis in four patients with cancer, neutropenia, and previous treatment with vancomycin. Eur J Clin Microbiol Infect Dis 1996:15:259-261.

8. Hiramatsu K, Hanaki H, Ino T, Yabuta $K$, Oguri T, Tenover FC. Methicillin-resistant Staphylococcus aureus clinical strain with reduced vancomycin susceptibility. I Antimicrob Chemother 1997;40:135-136.

9. Rotun SS, McMath V, Schoonmaker DJ, Maupin PS, Tenover FC, Hill $\mathrm{BC}$, et al. Staphylococcus aureus with reduced susceptibility to vancomycin isolated from a patient with fatal bacteremia. Emerging Infectious Diseases. In press.

10. Froggatt JW, Johnston JL, Galetto DW, Archer GL. Antimicrobial resistance in nosocomial isolates of Staphylococcus haemolyticus. Antimicrob Agents Chemother 1989;33:460-466.

11. Schwalbe RS, Stapleton JT, Gilligan PH. Emergence of vancomycin resistance in coagulase-negative staphylococci. $N \mathrm{Engl} \mathrm{J} \mathrm{Med}$ 1987;316:927-931.

12. Veach LA, Pfaller MA, Barrett M, Koontz FP, Wenzel RP. Vancomycin resistance in Staphylococcus haemolyticus causing colonization and bloodstream infection. J Clin Microbiol 1990;28:2064-2068.

13. Schwalbe RS, Ritz WJ, Verma PR, Barranco EA, Gilligan PH. Selection for vancomycin resistance in clinical isolates of Staphylococcus haemolyticus. I Infect Dis 1990;161:45-51.

14. Archer GL, Climo MW. Antimicrobial susceptibility of coagulase-negative staphylococci. Antimicrob Agents Chemother 1994;38:2231-2237.

15. Goldstein FW, Coutrot A, Sieffer A, Acar JF. Percentages and distribution of teicoplanin- and vancomycin-resistant strains among coagulasenegative staphylococci. Antimicrob Agents Chemother 1990;34:899-900.

16. Maugein J, Pellegrin JL, Brossard G, Fourche J, Leng B, Reiffers J. In vitro activities of vancomycin and teicoplanin against coagulase-negative staphylococci isolated from neutropenic patients. Antimicrob Agents Chemother 1990;34:901-903.

17. Bannerman TL, Wadiak DL, Kloos WE. Susceptibility of Staphylococcus species and subspecies to teicoplanin. Antimicrob Agents Chemother 1991;35:1919-1922.

18. Sieradzki K, Villari P, Tomasz A. Decreased susceptibilities to teicoplanin and vancomycin among coagulase-negative methicillin-resistant clinical isolates of staphylococci. Antimicrob Agents Chemother 1998;42:100-107

19. Gruneberg RN. Anti-gram-positive agents-what we have and what we would like. Drugs 1997;54(suppl) 6:29-38.

20. Kaatz GW, Seo SM, Dorman NJ, Lerner SA. Emergence of teicoplanin resistance during therapy of Staphylococcus aureus. I Infect Dis 1990;162:103-108.

21. Tenover FC, Lancaster MV, Hill BC, Steward CD, Stocker SA, Hancock $\mathrm{GA}$, et al. Characterization of staphylococci with reduced susceptibilities to vancomycin and other glycopeptides. J Clin Microbiol 1998;36: 1020-1027.

22. Shlaes DM, Shlaes JH, Vincent S, Etter L, Fey PD, Goering RV. Teicoplanin-resistant Staphylococcus aureus expresses a novel membrane protein and increases expression of penicillin-binding protein 2 complex. Antimicrob Agents Chemother 1993;37:2432-2437.

23. Shlaes DM, Shlaes JH. Teicoplanin selects for Staphylococcus aureus that is resistant to vancomycin. Clin Infect Dis 1995;20:1071-1073.

24. Moreira B, Boyle-Vavra S, deJonge BLM, Daum RS. Increased production of penicillin-binding protein 2 , increased detection of other penicillin-binding proteins, and decreased coagulase activity associated with glycopeptide resistance in Staphylococcus aureus. Antimicrob Agents Chemother 1997;41:1788-1793.

25. Sanyal D, Greenwood D. An electronmicroscope study of glycopeptide antibiotic-resistant strains of Staphylococcus epidermidis. J Med Microbiol 1993;39:204-210.

26. Kernodle DS, Barg NL, Kaiser AB. Low-level colonization of hospitalized patients with methicillin-resistant coagulase-negative staphylococci and emergence of the organism during surgical antimicrobial prophylaxis. Antimicrob Agents Chemother 1988;32:202-208. 


\section{Reducing the incidence of nosocomial UTI}

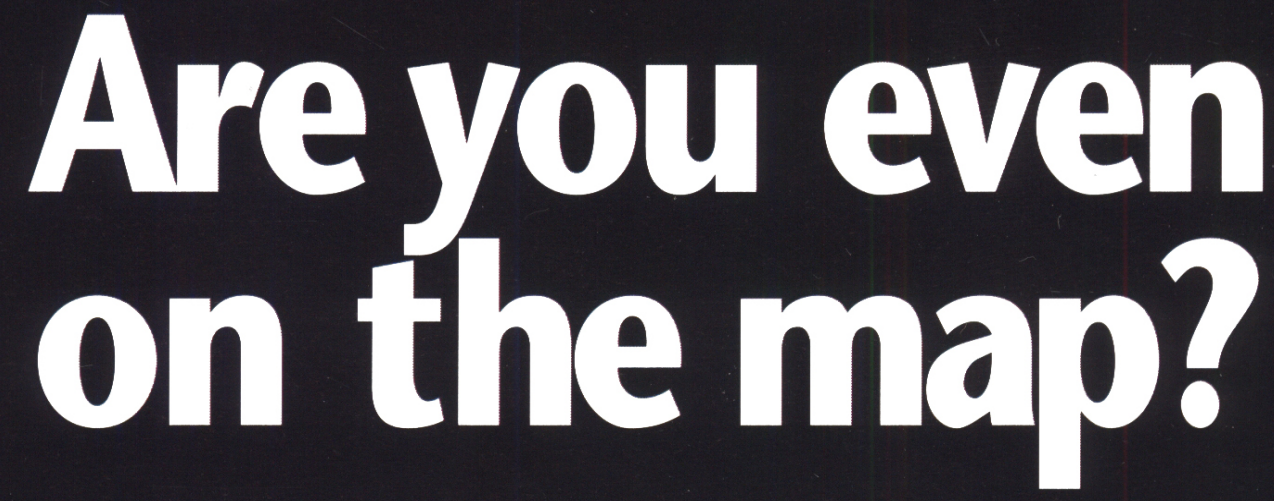

\section{The BARDEX ${ }^{\circledast}$ I.C. Foley Catheter can put you there.}

Each year, over 900,000 nosocomial urinary tract infections occur in hospitals throughout the U.S. - seriously impacting quality of care.

The silver and hydrogel-coated BARDEX I.C. Foley Catheter has been proven to reduce the incidence of NUTIs. The published examples noted below are just a few of the more than 600 success stories involving hospitals that significantly reduced infection rates and costs after converting to the BARDEX I.C. system.

For more information on the BARDEX I.C. Foley Catheter, contact your BARD Representative or call 1-800-526-4455. You can also visit our website at www.bardmedical.com.

Graduate

Philadelphia, PA

11,210 annual admissions $50 \%$

reduction in NUTI AUA-1998?
Kaiser Permanente Sunnyside Clackmas, OR

12,178 annual admissions

\section{$69 \%$}

reduction in NUTI APIC-1998:
St. Michael Health Care Ctr. Texarkana, TX 3,919 annual admissions

\section{$56 \%$}

reduction in NUTI

Poudre Valley

Fort Collins, $\mathrm{CO}$

13,264 annual admissions $53 \%$ reduction in NUTI APIC-1998:

APIC-1997
$\mathrm{U}$ of $\mathrm{W}$

Madison, WI

19,100 annual admissions

$25 \%$

reduction in NUTI

ICAAC-1998
UVA

Charlottesville, VA

27,116 annual admissions

$48 \%$

reduction in NUTI SHEA-1998

N. Broward Hospital Ft. Lauderdale, FL. 23,327 annual admissions $\mathbf{5 8} \%$ reduction in NUTI SHEA-1998

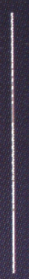

Wilson Memorial Hospital Wilson, NC 7,985 annual admissions $37 \%$ reduction in NUTI APIC-1996:
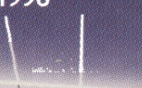

\section{Вस्तRRD}

Bard Medical Division

C.R. Bard, Inc.

8195 Industrial Boulevard

Covington, GA 30014

800-526-4455

References:

1. Data on file, BARD Medical Division, Covington, GA 